\title{
The Pursuit of Covid-19 Animal Models
}

Abdul Rahim R. ${ }^{a}$, Muhammad N. ${ }^{b}$

aDepartment of Basic Medical Science, Kulliyyah of Medicine, IIUM

bepartment of Pathology and Laboratory Medicine, Kulliyyah of Medicine, IIUM

\section{ABSTRACT}

Coronavirus disease 2019 (COVID-19) is a contagious disease instigated by a recently discovered coronavirus, the 2019 novel coronavirus (2019-nCoV). It has infected over 14 million people worldwide from all age groups. This virus is conveyed through big droplets produced during coughing and sneezing by the infected individuals. COVID-19 precipitates acute respiratory distress syndrome which leads to morbidity and mortality in severe cases. To date, there is neither an established specific antiviral treatment for COVID-19, nor vaccine available. Preventive measure of social distancing is deemed to be the most effective way to avoid the spread of the virus. Scientists are currently in search of appropriate COVID-19 animal models for use in research works related to pathogenesis, potential drug treatments and vaccine development. This review delivers a pithy overview of the currently available COVID-19 animal models published in the literatures.

KEYWORDS: COVID-19, animal models

\section{INTRODUCTION}

Coronaviruses are pleomorphic enveloped positive sense RNA viruses that are a genus in the Coronaviridae family and subfamily Coronavirinae. ${ }^{1,2}$ They range from $60 \mathrm{~nm}$ to $140 \mathrm{~nm}$ in diameter with spike-like projections on the surface (crown-like), from which the name coronavirus is derived. ${ }^{3}$ They cause infections in both humans and animals, and are commonly associated with self-limiting disease. ${ }^{4}$ However, severe acute respiratory diseases are known to occur with zoonotic coronaviruses infections which include the severe acute respiratory syndrome coronavirus (SARS-CoV) and Middle East respiratory syndrome coronavirus (MERS-CoV). Most recently discovered is the severe acute respiratory syndrome coronavirus 2 (SARS-CoV-2) or also known as the 2019 novel coronavirus (2019-nCoV) causing the coronavirus disease 2019 (COVID-19). ${ }^{5-7}$

The outbreaks of SARS-CoV in 2002 occurred in more than 30 countries with over 8098 cases and 774 deaths whilst those involving MERS-CoV in 2012 had

Corresponding Author:

Assoc. Prof. Dr. Roslina Abdul Rahim,

Department of Basic Medical Science,

Kulliyyah of Medicine,

International Islamic University Malaysia,

Tel No : +609-5704505

Email : roslinaar@iium.edu.my caused 779 deaths of the 2182 infected cases identified in 27 countries. $^{8}$ In contrast, the current SARS-CoV-2 infection is more widespread, involving over 215 countries and territories with over 14 million cases and approximately over 613,000 deaths as per $21^{\text {st }}$ of July $2020 .^{9}$ Although the SARS-CoV-2 infection demonstrates a lower rate of austere cases and case fatality compared to both SARS-CoV and MERS-CoV, ${ }^{8}$ it has a strikingly higher infectivity making it an appalling medical and social burden. Hence on the $11^{\text {th }}$ March 2020, the World Health Organization (WHO) declared it as a worldwide pandemic. $^{10}$

The SARS-CoV-2 infection (COVID-19) was first diagnosed in Wuhan City of Hubei Province, China ${ }^{11}$ and subsequently led to widespread infection in Italy. ${ }^{12}$ Italian scientists had earlier discovered traces of SARS-CoV-2 virus in the sewage system that were collected in Milan and Turin, although this did not imply that the main transmission chain that led to the development of the epidemic in Italy originated from these findings. ${ }^{13}$

The pathogenesis of COVID-19 is initiated by the receptor-mediated entry of SARS-CoV-2 into the host cells, where the viral $S$ protein receptor binding domain (RBD) of the SARS-CoV-2 binds to the human angiotensin converting enzyme 2 (ACE2) receptor or dipeptidyl peptidase 4 (DPP4) protein receptor. ${ }^{14}$ 
Within the cells, the virus is able to reproduce efficiently, mounting the cytopathogenesis and initiates the infection. ${ }^{8}$ The clinical features of COVID -19 range from an asymptomatic state to acute respiratory distress syndrome (ARDS) and multiple organ failure. ${ }^{15}$ Pneumonia, respiratory failure and death can occur in some patients by the end of the first week of infection. ${ }^{15,16}$ This is strongly associated with increase in a variety of inflammatory cytokines. ${ }^{16}$ Throat swab, nasopharyngeal swab, sputum, endotracheal aspirates and bronchoalveolar lavage are among the respiratory samples used to make the diagnosis. ${ }^{15,17}$ The reverse transcription polymerase chain reaction (RT-PCR) is the current standard diagnostic test for COVID-19. ${ }^{18}$

\section{COVID-19 Animal models}

Animal models have been used extensively in biological and biomedical science research to address various pertinent scientific questions for epochs. ${ }^{19}$ They are critical to the understanding of disease pathogenesis at molecular, cellular and physiological levels, development and assessment of novel treatments, new surgical techniques and also anaesthesia protocols. ${ }^{19,20}$ The findings in animal models played a strikingly important role that have led to a robust input in scientific knowledge and enhancement in life quality. ${ }^{19}$ Many Nobel prize winners in the field of Physiology and Medicine have used animal experimentation models for their innovations. $^{19}$

In relation to the current pandemic, among the main focus areas of research where animal models are extremely essential include the study of the viral infection and its pathogenesis which are closely intertwined with development and preclinical evaluation of a vaccine and antiviral drug. The availability of animal models that could provide consistent and reproducible results cannot be overemphasised in these circumstances. ${ }^{8}$

An animal model namely for the study of infectious diseases initiated by viruses has to fulfil a variety of characteristics, the first of which is that the animal model should mimic the human disease under investigation in terms of sharing the route of infection, severity of disease, typical clinical symptoms, host immune responses and has comparable levels of mortality and morbidity. ${ }^{8,21}$
Generally and also specific to SARS-CoV-2 infection, the viral entry and intracellular replication are critical in establishing a successful infection in an animal model $^{8}$ and there should be a correlation between the viral load and disease severity. ${ }^{21}$ Additionally the viral receptors and their distribution ought to be similar to that of humans. ${ }^{21}$ The speciesspecificity barrier is therefore an important criteria to be considered in selecting animal model for coronaviruses. ${ }^{8}$ For example, in mouse model it was found that the mouse dipeptidyl peptidase 4 (DPP4) protein receptors that is critical for the binding of viral S protein differs from that of the human ACE2 or DPP4 receptor. ${ }^{22}$ To overcome this problem, the human ACE2 expression is induced experimentally by knocking in or transfecting a human receptor gene into the mouse model, termed as humanized transgenic mouse model. ${ }^{23}$ In essence, the animal models must be judiciously assessed and selected to achieve the experimental targets. ${ }^{21}$

This review article provides a pithy overview of the most promising COVID-19 animal models based on their pathogenesis, histological findings, clinical symptoms similarities, the pros and cons of each model. This article will optimistically serve as a guide for scientists to choose the prime model for their research purposes.

\section{Ferret}

Ferrets are a well-known model for respiratory infections because of the similarity in their lung physiology to that of humans, where it can cough and sneeze. ${ }^{24-27}$ Ferrets infected with SARS-CoV-2 intranasally, showed viral replication limited to the nasal turbinate, soft palate and tonsils by day 4 post inoculation while no replication was detected in the lung lobes even after intratracheal inoculation of the virus. ${ }^{25}$ The viral RNA was also detected in the rectal swabs in smaller amount. ${ }^{25}$ SARS-CoV-2 gains entry into human body through ACE2 receptors expressed in the lungs, heart, kidneys and gastrointestinal tract, ${ }^{28-32}$ whilst in ferrets these receptors are found mainly on the type II pneumocytes and serous epithelial cells of the trachea-bronchial submucosal glands. ${ }^{33}$ As such it was concluded that the SARS-CoV -2 virus replicates more efficiently in the upper respiratory tract of ferrets and not in other organs. ${ }^{25}$

The SARS-CoV-2 virus infection in ferrets only increased their body temperature but other 
characteristic symptoms were absent. The virus cannot replicate to high levels, ${ }^{34}$ making the aforementioned factors as main disadvantages of this animal model. ${ }^{35}$ The infected ferrets however could spread the virus with a high transmission rate to adjacent cages, ${ }^{34}$ making them an excellent animal model for the study of transmission of SARS-CoV-2 $2^{36}$ and also for antiviral drug treatment and vaccine research works. ${ }^{25}$

\section{Cat}

In an experimental study that used this animal model, it was reported that sub-adult cats aged 6 to 9 months which were inoculated with SARS-CoV-2 via intranasal route, had viral replication in the nasal turbinate, soft palates, tonsils, tracheas, lungs, and small intestine at day 3 post inoculation. The viral RNA was also found in the faeces of these animals on days 3 and 5 post inoculation. An uninfected cat that was caged separately adjacent to the infected cat was eventually infected with the virus. This supports the airborne transmission route of this infection. ${ }^{25}$

Scientific studies on transmission of the disease were also carried out in juvenile cats aged between 70 to 100 days old. ${ }^{25}$ Massive lesions were observed in the nasal and mucosal epithelium of tracheas and lungs of the cats following intranasal inoculation of SARSCoV-2. This result indicates that the SARS-CoV-2 virus replicates efficiently in cats, especially the juvenile cats being more permissive via the airborne route. ${ }^{25}$ However, the epidemiological data in human shows that the elderly people are more vulnerable to SARSCoV-2 infection and had higher mortality rate. ${ }^{37}$

Cats may provide an expedient model system in studying the transmissibility of the virus. ${ }^{38}$ As most cats in Wuhan have been disclosed to be seropositive for SARS-CoV-2, ${ }^{39}$ the surveillance of SARS-CoV-2 in cats ought to be further explored in the efforts to contain COVID-19 in humans. ${ }^{25}$

\section{Dog}

The replication and conveyance of SARS-CoV-2 were examined in five 3-month old beagles inoculated intranasally with the virus. They were housed together with two uninoculated beagles in a room. The oropharyngeal and rectal swabs were taken post inoculation every 2 days for two weeks. The viral RNA was detected from the rectal swabs on days 2 and 4 post inoculation. The viral RNA was absent in all organs and tissues of the dogs. In the animals that were not inoculated, all swab results were negative. The SARS-CoV-2 antibody tests by enzyme immunoassay were also negative in the contact dogs. These results suggest that dogs have low susceptibility to infection by SARS-CoV-2. ${ }^{25}$

\section{Livestock animals}

Similar experimental studies performed in dogs were carried out in livestock animals such as chickens, ducks and pigs. ${ }^{25}$ The viral RNA was not noticed in any of the swabs from both the virally-inoculated and uninoculated animals. All animals were seronegative for SARS-CoV-2 on day 14 post inoculation. ${ }^{25}$ These animals are therefore not susceptible to SARS-CoV-2 infection. ${ }^{8,25}$ There are no reported investigations of SARS-CoV- 2 thus far in rabbits. ${ }^{8}$

\section{Non-human primate (NHP)}

\section{Cynomolgus macaque}

Cynomolgus macaque is one of the most commonly used NHPs in biomedical research due to its genome being highly homologous to human and the relative ease of carrying out experiments with this animal model. Published findings have shown that cynomolgus macaques are susceptible to SARS-CoV-2 infection, have a prolonged period of viral shedding and exhibit COVID-19-like disease. It has also been proven that SARS-CoV-2 reproduces efficiently in the epithelial cells throughout the respiratory tract. The replication in the upper respiratory tract fits with efficient transmission between hosts, while the replication in the lower respiratory tract fits with the progression of lung disease. ${ }^{40}$

In an experimental study, young (aged 4-5 years) and old adults cynomolgus macaques (15-20 years of age) were inoculated with SARS-CoV-2 strain via a combination of intratracheal and intranasal routes. ${ }^{40}$ The viral RNA was detected to peak by days 2 and 4 post inoculation in the young and aged animals respectively. The viral replication was spotted around the nasal cavity, trachea, bronchi and lung lobes with the utmost levels of expression being in the lungs. ${ }^{40}$ The RNA level was higher in the nasal swabs of aged as compared to young animals. There was prolonged shedding of the SARS-CoV-2 in the upper respiratory tract of this animal. ${ }^{40}$ 
There were no clinical signs or weight loss seen septum, degeneration of epithelial lining and in all these animals by day 14 post inoculation except exudation within the alveoli cavities. Essentially, the for one old-aged macaque that had serous nasal inoculation of the older rhesus macaques with SARSdischarge at day 14 post inoculation. ${ }^{40}$ Amongst CoV- 2 had caused a more serious form of interstitial the histopathological changes observed were pneumonia. ${ }^{45}$

consolidation changes of lung tissue, alveoli hyaline

membrane formation, diffuse alveoli damage, The Rhesus macaque can be a promising model to type II pneumocyte hyperplasia and inflammatory study the efficiency of vaccines as the immune changes in the respiratory tract. ${ }^{40}$ Injury to type I responses in monkeys exhibit similarity to that of the pneumocytes can result in pulmonary oedema, humans. ${ }^{36}$ However the clinical signs documented and formation of hyaline membranes ${ }^{40,41}$ which may were transient hence posing a disadvantage for this explain why hyaline membrane formation is a non-human primate model. ${ }^{35}$

hallmark for COVID-19. ${ }^{40,42,43}$ These changes coupled with expression of SARS-CoV-2 antigen by the types I and II pneumocytes and lung tissues provide strong supporting evidence that the SARS-CoV-2 infection caused the lesions. ${ }^{40}$ This study contributed a novel animal model which will be crucial in the assessment and licensure of preventive and therapeutic approaches against SARS-CoV-2 infection for use in humans. ${ }^{40}$

Although the advantage of this model is that the viral titre remains for longer period of time with development of characteristic histopathological changes, the absence of overt clinical signs is deemed to be a disadvantage. ${ }^{35}$

\section{African Green Monkey}

African Green monkeys (AGMs) were also used as COVID-19 research animal model. These monkeys were inoculated with intratracheal and intranasal low doses of SARS-CoV-2 virus that was isolated from the first case in Italy. There were no overt clinical signs observed in these monkeys except for decreased in appetite and transient fever. Histological findings at day 5 post inoculation revealed marked pneumonia characterised by pulmonary consolidation and haemorrhage. The inflammatory mediator profiles were similar to that seen in the human immune response to SARS-CoV-2. High viral loads were spotted in mucosal samples of

\section{Rhesus Macaque}

Rhesus macaque is another non-human primate animal model for COVID-19 that is a closer model to humans. ${ }^{36}$ The macaque also has the same amino acid residues of human ACE2. ${ }^{44} \mathrm{~A}$ study was conducted which also used both young (age 3 to 5 years) and oldaged (15 years) macaques where all were inoculated with SARS-CoV-2 via intratracheal route. The body weight was reduced in all macaques except in one young macaque. There was no changed in the body temperature. The viral RNA was detected in the nose, pharynx, and crissum of all the macaques. The viral load peaked on day 3 post inoculation in both the young and old macaques, particularly in the respiratory tract system. ${ }^{45}$ all animals including faeces of several animals on day 15 post inoculation. ${ }^{46}$

This model is considered a gold standard animal model for the study of pathogenesis of COVID-19 in comparison to other NHP models predominantly as the model requires a lower dose of inoculation to exert the effects. The drawback of this model however is that the animals did not develop debilitating clinical signs and symptoms as in the human counterpart. This model is costly and difficult to handle the animals. ${ }^{35}$

\section{Golden Syrian Hamster}

A recent animal model of COVID-19 was developed using the Golden Syrian hamster (Mesocricetus

The chest $\mathrm{x}$-rays revealed ground-glass opacity which auratus) to study the pathogenesis, transmission and was more prominent in the older macaque. passive immunization effects of COVID-19. ${ }^{47}$ The in Histologically both groups of macaque exhibited silico structural analysis hypothesises that the pneumonic changes with more severe lesions being hamster ACE2 will bind to SARS-CoV-2 spike observed in the old-aged macaques. The changes glycoprotein receptor-binding domain at high binding include inflammatory cells infiltrate in the alveoli affinity. The ACE2 proteins of the hamster are highly interstitium and cavities, thickening of alveolar similar to that of the human. ${ }^{36}$ 
A study that utilized this animal model inoculated with SARS-CoV-2 intranasally revealed that the animals became symptomatic with features that include ruffled furs, hunched back posture, lethargy and dyspnoea on day 2 post inoculation. ${ }^{47}$ The histopathological changes ranged from diffuse alveolar damage with extensive apoptosis to proliferative phase of tissue repair in the later stage. The lymphoid organs also exhibited atrophic changes which were attributed to cytokine activation that occurred within the first week post inoculation. Naïve hamsters which were housed in the same cage as the infected hamsters also developed similar pathology. All infected hamsters ameliorated and developed a mean serum neutralising antibody titre of $\geq 1: 427$ by day 14 post inoculation with a significant decrease in the lung viral load. There was no mortality reported throughout the study. ${ }^{47}$ This model is deemed to be appropriate for studying the pathogenesis, transmission, immune responses, treatments and vaccines for COVID-19. ${ }^{36,47}$

\section{Human Angiotensin-converting enzyme 2 (hACE2) transgenic mice}

In a study, both male and female 11-month-old hACE2 transgenic mice, were inoculated intranasally with 2019-nCoV stock virus. The transgenic mice had significant reduction in body weight. The lung and liver tissues showed the presence of the virus. The histopathological examination demonstrated that the bronchioles, blood vessels, alveolar interstitium and the alveolar epithelial cells of the transgenic mice were infected with 2019-nCoV. ${ }^{48}$ However there were no significant histopathological lesions or viral antigens observed in the myocardium, liver, spleen, kidney, cerebrum, intestine and testis. The pneumonia became mild with only focal lesions at day 7 post inoculation, signifying a non-lethal and selflimiting infection course. ${ }^{48}$ Thus; this model does not mimic the austere and lethal cases of COVID-19 in humans. ${ }^{36}$

\section{Advantages and disadvantages of animal models}

Animals are excellent research subjects as they have biological similarities to that of humans and are susceptible to diseases. ${ }^{36}$ In contrast to humans, the progress of a disease can be studied and documented in animal models due to their shorter life-cycles and under a controlled environment. ${ }^{36}$ COVID-19 research works involving animal model development would allow for the understanding of the disease mechanisms prior to the development of vaccines and other therapeutic modalities namely drugs. ${ }^{49}$

However, some of the results generated from animal studies were unable to be translated to humans, such as human trial of COVID-19 drug Remdesivir which yielded better outcomes in rhesus macaque model. ${ }^{50}$ Animal studies have been shown to not predict the outcomes in humans despite them sharing biological similarities since the final response to disease depends partly on the epigenome and environmental variations. ${ }^{36}$

\section{CONCLUSION}

There are many animal models to date that can be used to study the SARS-CoV-2 infection. Ferrets, nonhuman primates (cynomolgus macaques, rhesus macaques and African green monkey), Golden Syrian hamster and hACE2 transgenic mice are deemed to be the most promising animal models in COVID-19 research works, namely in areas related to pathogenesis, anti-viral treatment and vaccine development. Livestock animal such as chickens, ducks and pigs are considered to be not suitable models for COVID-19 due to its insusceptibility to SARS-CoV-2 infection.

All models have their pros and cons based on the findings of published studies. Finally, it is important to emphasise that experimental outcomes attained in animal studies were not necessarily confirmed in further human studies despite large similarities between human and the chosen animals. This is because there are some differences between a given animal species and humans.

\section{CONFLICT OF INTERESTS}

All the authors declare no financial conflict of interests upon the preparation of this manuscript.

\section{REFERENCE}

1. Woo PC, Huang Y, Lau SK., Yuen KY. Coronavirus genomics and bioinformatics analysis. Viruses, 2 (8):1804-1820 (2010).

2. Abebe EC, Dejenie TA, Shiferaw MY, Malik T. The newly emerged COVID-19 disease: a systemic review. Virol J 17:96 (2020).

3. Richman DD, Whitley RJ, Hayden FG. Clinical virology. John Wiley \& Sons, (2016). 
4. Graham RL, Donaldson EF, Baric RS. A decade after SARS: strategies for controlling emerging coronaviruses. Nature Reviews Microbiology. 11 (12):836-48 (2013).

5. Ksiazek TG, Erdman D, Goldsmith CS, Zaki SR, Peret $\mathrm{T}$, et al. A novel coronavirus associated with severe acute respiratory syndrome. New England journal of medicine. 348(20):1953-66 (2003).

6. Assiri A, Al-Tawfiq JA, Al-Rabeeah AA, Al-Rabiah FA, Al-Hajjar S, et al. Epidemiological, demographic, and clinical characteristics of 47 cases of Middle East respiratory syndrome coronavirus disease from Saudi Arabia: a descriptive study. The Lancet infectious diseases. 13(9):752-61 (2013).

7. Wang C, Horby PW, Hayden FG, Gao GF. A novel coronavirus outbreak of global health concern. The Lancet. 395(10223):470-3 (2020).

8. Yuan L, Tang $\mathrm{Q}$, Cheng $\mathrm{T}$, Xia N. Animal models for emerging coronavirus: progress and new insights. Emerging Microbes \& Infections. 9:1-26 (2020).

9. Coronavirus Update live. https:// www.worldometers.info/coronavirus/. Accessed on $21^{\text {st }}$ July 2020.

10. World Health Organization. WHO DirectorGeneral's opening remarks at the media briefing on COVID-19, 11 March 2020. https://

www.who.int/dg/speeches/detail/who-director -general-s-opening-remarks-at-the-media briefing-on-covid-19---11-march-2020. Accessed on $11^{\text {th }}$ July 2020.

11. Huang $C$, Wang $Y$, Li X, Ren $L$, Zhao J, et al. Clinical features of patients infected with 2019 novel coronavirus in Wuhan, China. The Lancet. 395(10223):497-506 (2020).

12. Chen J, Lu H, Melino G, Boccia S, Piacentini M, et al. COVID-19 infection: the China and Italy perspectives. Cell Death \& Disease. 8;11(6):1-7 (2020).

13. Sham N. Water samples suggest COVID-19 present in Italy before Chinese cases. https: / / newseu.cgtn.com/news/2020-06-20/Watersamples-suggest-COVID-19-present-in-Italybefore-Chinese-cases-RtTMEUvgcg/index.html. Accessed on $27^{\text {th }}$ June 2020.

14. Zhou $P$, Yang $X L$, Wang $X G$, Hu B, Zhang $L$, et al. A pneumonia outbreak associated with a new coronavirus of probable bat origin. Nature. 579 (7798):270-3 (2020).
15. Singhal T. A review of coronavirus disease-2019 (COVID-19). The Indian Journal of Pediatrics. 13:1-6 (2020).

16. Chen N, Zhou M, Dong X, Qu J, Gong F, et al. Epidemiological and clinical characteristics of 99 cases of 2019 novel coronavirus pneumonia in Wuhan, China: a descriptive study. The Lancet. 395(10223):507-13 (2020).

17. Huang $P$, Liu $T$, Huang $L$, Liu $H$, Lei $M$, et al. Use of chest CT in combination with negative RT-PCR assay for the 2019 novel coronavirus but high clinical suspicion. Radiology. 295(1):22-3 (2020).

18. Corman VM, Landt O, Kaiser M. Detection of 2019 novel coronavirus (2019-nCoV) by real-time RTPCR. Euro Surveill. 25:23-30 (2020).

19. Andersen ML, Winter LMF. Animal models in biological and biomedical research experimental and ethical concerns. An Acad Bras Cienc. 91(suppl 1):e20170238 (2019).

20. Barré-Sinoussi F, Montagutelli X. Animal models are essential to biological research: issues and perspectives. Future science.1(4) (2015).

21. Gretebeck LM, Subbarao K. Animal models for SARS and MERS coronaviruses. Current opinion in virology.13:123-9 (2015).

22. Cockrell AS, Peck KM, Yount BL, Agnihothram SS, Scobey $T$, et al. Mouse dipeptidyl peptidase 4 is not a functional receptor for Middle East respiratory syndrome coronavirus infection. Journal of Virology. 1;88(9):5195-9 (2014).

23. Tseng $C T$, Huang $C$, Newman $P$, Wang $N$, Narayanan K, et al. Severe acute respiratory syndrome coronavirus infection of mice transgenic for the human Angiotensin-converting enzyme 2 virus receptor. Journal of virology. 81 (3):1162-73 (2007).

24. Callaway, E. Monkeys and mice enlisted to fight corona virus. Nature. 579:183 (2020).

25. Shi J, Wen Z, Zhong G, Yang H, Wang C, et al. Susceptibility of ferrets, cats, dogs, and other domesticated animals to SARS-coronavirus 2. Science. 368(6494):1016-1020. (2020).

26. Cameron MJ, Kelvin AA, Leon AJ, Cameron CM, Ran $L$, et al. Lack of innate interferon responses during SARS coronavirus infection in a vaccination and reinfection ferret model. PLOS one. 24;7(9):e45842 (2012).

27. Enkirch T, Von Messling V. Ferret models of viral pathogenesis. Virology. 479:259-70 (2015).

28. Rabi FA, Al Zoubi MS, Kasasbeh GA, Salameh DM, Al-Nasser AD. SARS-CoV-2 and Coronavirus 
Disease 2019: What we know so far. Pathogens. 3, 231 (2020).

29. Yan R, Zhang Y, Li Y, Xia L, Guo Y, et al. Structural basis for the recognition of SARS-CoV2 by full-length human ACE2. Science. 367 (6485), 1444-8 (2020).

30. Donoghue M, Hsieh F, Baronas E, Godbout K, Gosselin $M$, et al. A novel angiotensinconverting enzyme-related carboxypeptidase (ACE2) converts angiotensin I to angiotensin 1-9. Circ. Res. 87:E1-E9 (2000).

31. Zhang $H$, Kang Z, Gong $H$, Xu D, Wang J, et al. The digestive system is a potential route of 2019 -nCov infection: a bioinformatics analysis based on single-cell transcriptomes. BioRxiv. (2020).

32. Zhao Y, Zhao Z, Wang Y, Zhou Y, Ma Y, et al. Single-cell RNA expression profiling of ACE2, the putative receptor of Wuhan 2019-nCov. BioRxiv (2020).

33. Van den Brand JM, Haagmans BL, Leijten $L$, van Riel D, Martina BE, et al. Pathology of experimental SARS coronavirus infection in cats and ferrets. Veterinary pathology. 45(4), 551-62 (2008).

34. Kim YI, Kim SG, Kim SM, Kim EH, Park SJ, et al. Infection and rapid transmission of SARS-CoV-2 in ferrets. Cell host \& microbe. 27(5): 704-709 (2020).

35. Singh A, Singh RS, Sarma P, Batra G, Joshi R, et al. A Comprehensive Review of Animal Models for Coronaviruses: SARS-CoV-2, SARS-CoV, and MERS-CoV. Virologica Sinica. 30:1-5 (2020).

36. Deb B, Shah H, Goel S. Current global vaccine and drug efforts against COVID-19: Pros and cons of bypassing animal trials. Journal of Biosciences. 45(1):1-10 (2020).

37. Chan JF, Yuan S, Kok KH, To KK, Chu H, et al. A familial cluster of pneumonia associated with the 2019 novel coronavirus indicating person-toperson transmission: a study of a family cluster. The Lancet. 395(10223):514-23 (2020).

38. Lakdawala SS, Menachery VD. The search for a COVID-19 animal model. Science. 368(6494):942 -3 (2020).

39. Zhang Q, Zhang H, Huang K, Yang Y, Hui X, et al. SARS-CoV-2 neutralizing serum antibodies in cats: a serological investigation. BioRxiv.1-12 (2020).

40. Rockx B, Kuiken T, Herfst S, Bestebroer T, Lamers MM, et al. Comparative pathogenesis of COVID-19, MERS, and SARS in a nonhuman primate model. Science. 368(6494):1012-15 (2020).

41. Ware LB, Matthay MA. The acute respiratory distress syndrome. New England Journal of Medicine. 342(18):1334-49 (2000).

42. Tian S, Hu W, Niu L, Liu H, Xu H, et al. Pulmonary pathology of early phase 2019 novel coronavirus (COVID-19) pneumonia in two patients with lung cancer. Journal of Thoracic Oncology. 15(5): 700-704 (2020).

43. Kuiken T, Fouchier RA, Schutten M, Rimmelzwaan GF, Van Amerongen G, et al Newly discovered coronavirus as the primary cause of severe acute respiratory syndrome. The Lancet. 362(9380): 263-70 (2003).

44. Melin AD, Janiak MC, Marrone III F, Arora PS, Higham JP. Comparative ACE2 variation and primate COVID-19 risk. BioRxiv. (2020)

45. Yu P, Qi F, Xu Y, Li F, Liu P, et al. Age-related rhesus macaque models of COVID-19. Animal Models and Experimental Medicine. 3:93-97 (2020).

46. Woolsey C, Borisevich V, Prasad AN, Agans KN, Deer DJ, et al. Establishment of an African green monkey model for COVID-19. BioRxiv. (2020).

47. Chan JF, Zhang AJ, Yuan S, Poon VK, Chan CC, et al. Simulation of the clinical and pathological manifestations of Coronavirus Disease 2019 (COVID-19) in golden Syrian hamster model: implications for disease pathogenesis and transmissibility. Clinical Infectious Diseases. 1-51(2020).

48. Bao L, Deng W, Huang B, Gao H, Ren L, et al. The pathogenicity of 2019 novel coronavirus in hACE2 transgenic mice. Nature. 020-2312y (2020).

49. Swearengen JR. Choosing the right animal model for infectious disease research. Animal models and experimental medicine. 1(2):100-8 (2018).

50. Covid-19 drug Remdesivir fails in human trial. New Straits Times (Online). Accessed on $27^{\text {th }}$ June 2020. https: / /www.nst.com.my/world/ world/ 2020/04/587048/covid-19-drugremdesivir-fails-human-trial 